\title{
CALCUL ÉLECTROMAGNÉTIQUE DE MICRO ET NANOSTRUCTURES PHOTONIQUES PÉRIODIQUES
}

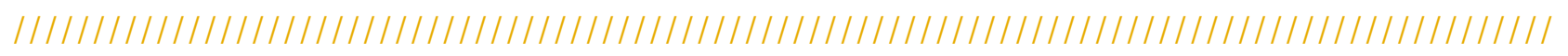

Mondher BESBES, Jean-Paul HUGONIN, Christophe SAUVAN

Université Paris-Saclay, Institut d'Optique Graduate School, CNRS, Laboratoire Charles Fabry, 91127 Palaiseau, France
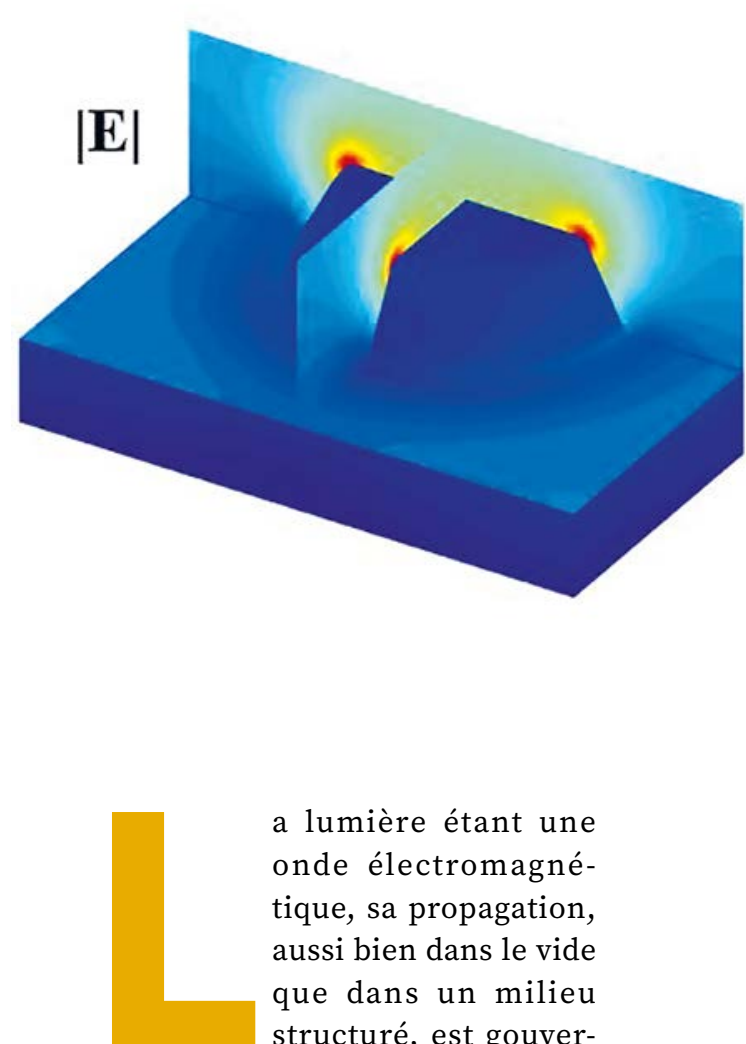

a lumière étant une onde électromagnétique, sa propagation, aussi bien dans le vide que dans un milieu structuré, est gouvernée par les équations de Maxwell. Jusqu'à l'avènement des micro et nanotechnologies, il était plutôt rare que l'opticien ait besoin de résoudre ces équations dans toute leur généralité. Comme la taille des structures considérées était nettement plus grande ou plus petite que la longueur d'onde, la propagation, la diffraction et la diffusion de la lumière pouvaient être décrites de façon précise en introduisant des approximations conduisant par
Depuis plus de vingt ans, la photonique bénéficie à la fois des techniques de micro et nanofabrication développées par l'industrie de la microélectronique, et des progrès des processus de synthèse chimique et biochimique. C'est ainsi que les composants optiques et optoélectroniques incluent des micro et nanostructures à la géométrie de plus en plus complexe et de mieux en mieux contrôlée. Cet essor de la nanophotonique s'est accompagné d'un accroissement des besoins en calcul électromagnétique des propriétés optiques de micro et nanostructures.

\section{https://doi.org/10.1051/photon/202010034}

Article publié en accès libre sous les conditions définies par la licence Creative Commons Attribution License CC-BY (http://creativecommons.org/licenses/by/4.0), qui autorise sans restrictions l'utilisation, la diffusion, et la reproduction sur quelque support que ce soit, sous réserve de citation correcte de la publication originale. exemple à l'optique géométrique, à l'optique de Fourier ou aux théories d'homogénéisation.

Les premiers travaux sur l'élaboration de méthodes numériques pour résoudre les équations de Maxwell ont été réalisés pour les radiofréquences et les micro-ondes, en parallèle des premiers développements de l'informatique. Les opticiens se sont tournés vers les méthodes numériques dans les années 60 puis 70 pour l'étude des guides d'onde, des réseaux de diffraction pour la spectroscopie ou des réseaux coupleurs pour l'optique intégrée. Le développement et la démocratisation des méthodes numériques sont ensuite allés de pair avec l'essor de l'informatique et l'accroissement considérable des puissances de calcul. La simulation numérique occupe aujourd'hui une place importante dans la phase de conception de composants photoniques. Elle apporte une aide majeure à l'opticien pour concevoir et optimiser de nouvelles micro et nanostructures aux propriétés optiques originales en évitant de multiples fabrications coûteuses.

Même si la nanophotonique ne s'intéresse pas uniquement à des structures périodiques, celles-ci occupent une place importante dans la réalisation de composants. Les structures périodiques utilisées en nanophotonique ont en 
général une période sub-longueur d'onde. Dans ce cas, parmi tous les ordres de diffraction, seul l'ordre 0 se propage en champ lointain, comme expliqué à la figure 1. Les autres ordres de diffraction sont des ondes évanescentes cantonnées au champ proche.

\section{DE NOMBREUSES}

\section{MÉTHODES NUMÉRIQUES...}

Il existe actuellement de nombreuses méthodes numériques permettant de résoudre les équations de Maxwell. Certaines sont basées sur des algorithmes génériques de résolution d'équations aux dérivées partielles (différences finies, éléments finis...) et permettent de traiter n'importe quelle géométrie alors que d'autres sont au contraire plus spécifiques et ne s'appliquent qu'à un seul type de problème. Il est donc difficile de dresser une liste exhaustive et notre objectif est plutôt de donner au lecteur quelques critères permettant de différencier les méthodes entre elles.

Les méthodes temporelles résolvent les équations de Maxwell en fonction des variables d'espace et de temps et permettent de suivre l'évolution du champ électromagnétique au cours du temps quel que soit son contenu fréquentiel. Les méthodes diffèrent par les moyens choisis pour la discrétisation spatiale ainsi que par la façon de réaliser l'intégration temporelle. Par exemple, la méthode FDTD (finite-difference time-domain), une des méthodes numériques les plus répandues, discrétise les trois directions de l'espace en remplaçant les dérivées spatiales par des différences finies [1].

Les méthodes fréquentielles considèrent quant à elles des champs électromagnétiques monochromatiques. Le temps disparaît des équations au profit de la fréquence de l'onde et la résolution se fait fréquence par fréquence en fonction des seules variables d'espace. Là encore, de nombreuses possibilités existent. Une première famille regroupe les méthodes basées sur la discrétisation des trois dimensions de l'espace comme les différences finies ou la méthode des éléments finis FEM (finite element method) [2]. Grâce à l'utilisation de différents éléments hiérarchiques (tétraèdres, pyramides...), la FEM possède l'avantage de pouvoir traiter des formes complexes sans avoir besoin d'approcher la géométrie par des marches d'escalier. Cependant, elle nécessite une discrétisation de tout l'espace qui s'avère vite très gourmande en mémoire vive et en temps de calcul pour des problèmes tridimensionnels.

Une autre famille de méthodes fréquentielles exploite la périodicité du système en développant les champs électromagnétiques en séries de Fourier. Autrement dit, une ou deux dimensions spatiales sont discrétisées dans l'espace réciproque. Dans la dimension restante (celle suivant laquelle la structure ne présente pas de périodicité), les équations peuvent être intégrées soit numériquement, c'est le cas de la méthode différentielle [3], soit analytiquement si la distribution d'indice de réfraction est invariante dans cette direction. C'est le cas de la méthode modale de Fourier (acronyme anglais FMM), également appelée rigorous coupled-wave analysis (RCWA). Enfin, il existe également d'autres méthodes modales qui n'utilisent pas les séries de Fourier et discrétisent les

\section{- SPECTROGON}

State of the art products

\section{Filtres Interférentiels}

De 200 á 15000 nm

- Passe-bande

- Passe-haut

- Passe-bas

- Large bande

- Densité neutre

- Disponible en stock

\section{Réseaux Holographiques}

De 150 á $2000 \mathrm{~nm}$

- Compression d'impulsion

- Télècom

- Accordabilité spectrale

- Monochromateurs

- Spectroscopie

- Disponible en stock 
directions de périodicité différemment. En intégrant les équations de Maxwell analytiquement dans une direction, les méthodes modales permettent généralement un gain en temps de calcul par rapport à des méthodes qui discrétisent tout l'espace. En utilisant explicitement les modes de la structure ainsi que leurs couplages aux interfaces, elles permettent également une compréhension physique approfondie. En contrepartie, ces méthodes ne sont pas toujours bien adaptées aux géométries complexes, notamment les formes courbes.

\section{LOI DES RÉSEAUX}

Un réseau de période $d$ est formé par une distribution quelconque d'indice de réfraction qui se répète périodiquement. Le schéma du bas montre un réseau déposé sur un substrat d'indice $n_{b}$ et éclairé depuis le superstrat d'indice $n_{h}$ par une onde plane dont la direction forme un angle $\boldsymbol{\theta}_{i n c}$ avec la verticale (flèches rouges). Le réseau diffracte l'onde incidente en un ensemble discret d'ondes planes en réflexion (ordres de diffraction réfléchis) et en transmission (ordres de diffraction transmis). La formule des réseaux permet de calculer la composante parallèle à la surface du vecteur d'onde de l'ordre de diffraction numéro

$p, k_{/ /}^{p}=\frac{2 \pi}{\lambda}\left|n_{h} \sin \Theta_{i n c}+p \frac{\lambda}{d}\right|$. Seuls les ordres réfléchis tels que $\left|k_{/ /}^{p}\right|<\frac{2 \pi}{\lambda} n_{h}$ et les ordres transmis tels que $\left|k_{/ /}^{p}\right|<\frac{2 \pi}{\lambda} n_{b}$ sont des ondes planes propagatives.

Ils sont représentés par des flèches bleues. Pour l'exemple de gauche il y a deux ordres diffractés en haut (réflexion) et trois en bas (transmission). Sur l'exemple de droite il n'y a qu'un seul ordre en haut et un seul ordre en bas (ordre $p=0$ ) car la période est plus petite. Il s'agit d'un réseau sub-longueur d'onde. Tous les autres ordres de diffraction sont des ondes évanescentes qui ne transportent pas d'énergie en champ lointain. La formule des réseaux ne contient aucune approximation ; elle est une conséquence directe du théorème de Floquet-Bloch et est valable quelle que soit la géométrie du réseau. En revanche, les amplitudes des ordres de diffraction dépendent de la géométrie et leur calcul nécessite l'utilisation de codes numériques.

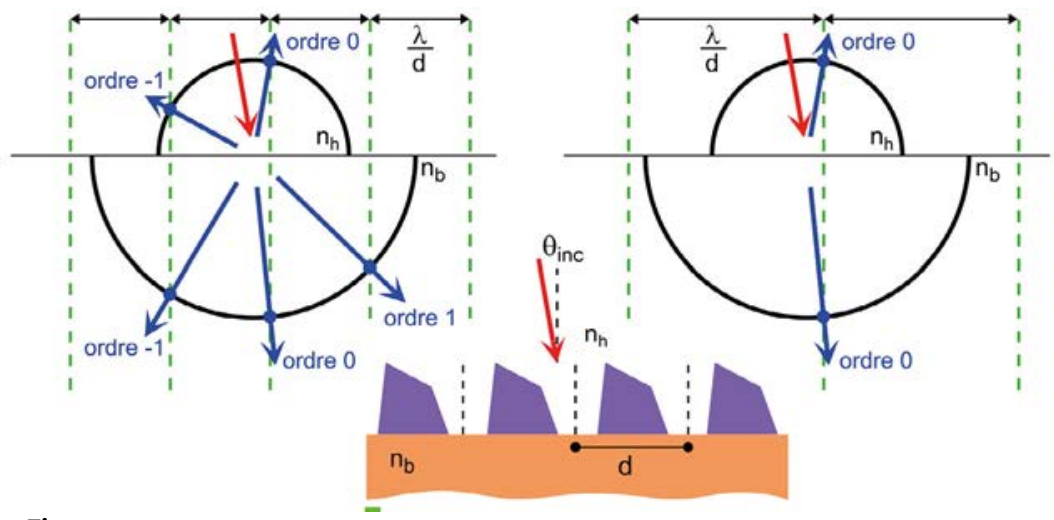

Figure 1.

La figure illustre la formule des réseaux par une construction géométrique similaire à la construction des lois de Descartes pour un dioptre plan.

\section{... MAIS UNE \\ DIFFICULTÉ COMMUNE}

Chaque méthode possède ses propres spécificités, ses avantages et ses inconvénients, mais toutes font face au même défi majeur : calculer le champ électromagnétique au voisinage d'une interface entre un matériau diélectrique et un matériau au caractère métallique prononcé, c'est-à-dire dont la permittivité relative présente une partie réelle négative et un module beaucoup plus grand que l'unité. C'est le cas des métaux nobles (or, argent) aux fréquences plus petites que leur fréquence plasma (du visible à l'infrarouge lointain) mais également des semiconducteurs dopés dans l'infrarouge. Des structures métal-diélectrique créent des contrastes de permittivité extrêmement grands qui génèrent des difficultés numériques. Tout d'abord, certaines composantes du champ présentent une très forte discontinuité au niveau de l'interface. En outre, le champ décroit extrêmement vite de part et d'autre de l'interface, avec des longueurs caractéristiques de décroissance très petites par rapport à la longueur d'onde. À cela vient s'ajouter le fait que le champ électromagnétique présente des singularités au niveau des arrêtes [4].

Pour résoudre ces difficultés numériques, les méthodes de type éléments finis utilisent des techniques de maillage adaptatif qui permettent de discrétiser l'espace plus finement dans ces zones où le champ varie rapidement. Les méthodes qui développent le champ électromagnétique en séries de Fourier semblent de prime abord plus démunies face au problème. En réalité, il est possible d'utiliser des transformations de coordonnées pour réaliser l'équivalent d'un « zoom » autour des interfaces, de façon à régulariser les singularités et à rendre les champs plus « facilement » développables en série de Fourier. Cette amélioration de la FMM est illustrée à la figure 2. 
(d)
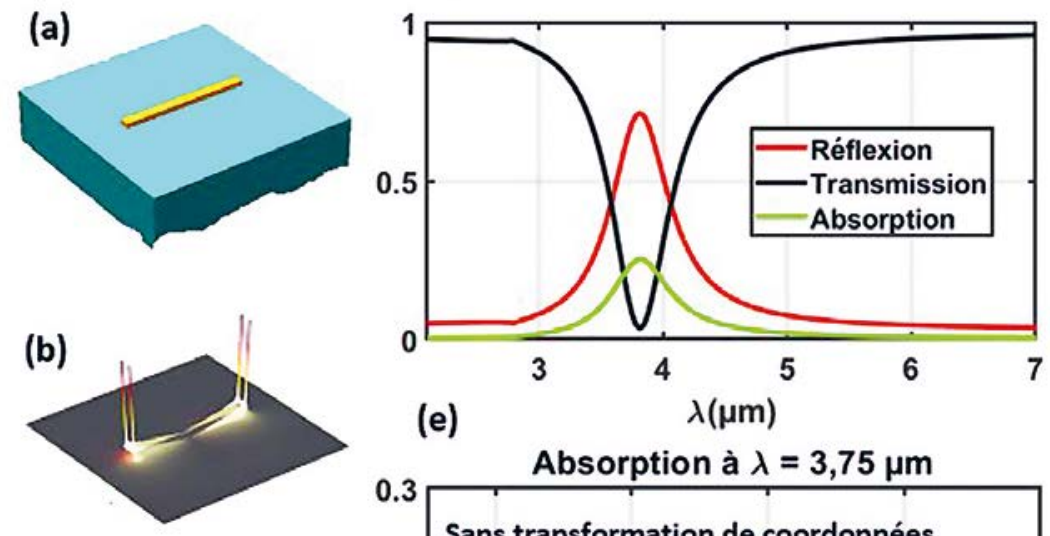

Absorption à $\lambda=3,75 \mu \mathrm{m}$
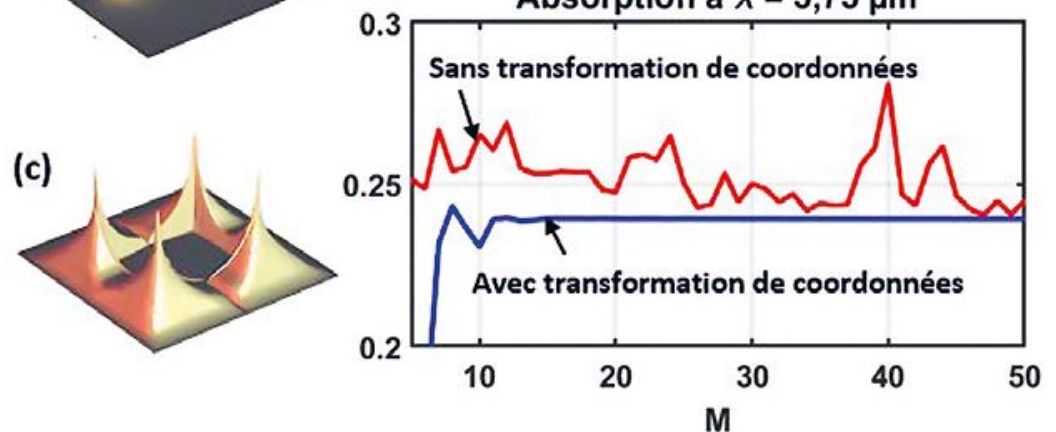

\section{LA MÉTHODE MODALE \\ DE FOURIER (FMM)}

La FMM est une méthode modale fréquentielle qui permet de résoudre le problème de la diffraction par une structure périodique. Cette méthode a été proposée initialement vers la fin des années 70 pour l'analyse électromagnétique des réseaux de diffraction [5]. Elle a également été utilisée dans le domaine des cristaux photoniques pour le calcul des diagrammes de bande sous le nom de méthode des ondes planes. Dans le cas de réseaux profonds ou de structures multicouches, l'utilisation du formalisme des matrices $\mathrm{S}$ (scattering matrices) permet d'obtenir une très bonne stabilité numérique.

Si la FMM souffrait à ses débuts d'une mauvaise convergence dans certains cas, ses performances numériques ont beaucoup progressé dans la deuxième moitié des années 90. Les problèmes rencontrés initialement ont été résolus [6-8] et la FMM est aujourd'hui une méthode numérique fiable et largement utilisée. Il existe plusieurs logiciels commerciaux incluant la FMM parmi d'autres méthodes (RSoft, Photon Design...) ainsi que de $\bullet \bullet \bullet$
Figure 2.

(a) Schéma d'une période $(2 \mu \mathrm{m} \times 2 \mu \mathrm{m})$ d'un réseau de bâtonnets d'or (dimensions $1,2 \mu \mathrm{m} \times 100 \mathrm{~nm} \times 50 \mathrm{~nm}$ ) déposés sur un substrat de silice (indice

1,4). (b) Distribution du module du champ électrique dans le plan $(x, y)$ situé $25 \mathrm{~nm}$ au-dessus du substrat. Le champ est exalté et varie extrêmement rapidement sur les bords du métal. (c) Distribution du module du champ électrique avec la transformation de coordonnées. Celle-ci permet de dilater les variations du champ. (d) Spectres de réflexion, de transmission et d'absorption. (e) Convergence du calcul FMM en fonction du nombre $M$ de termes de Fourier (séries de -M à +M) avec (courbe bleue) et sans (courbe rouge) transformation de coordonnées pour une longueur d'onde de $3,75 \mu \mathrm{m}$. Dans les calculs, la permittivité relative de l'or est donnée par un modèle de Drude $\varepsilon_{\text {or }}(\omega)=1-\omega_{p}^{2} /\left(\omega^{2}+i \omega \gamma\right)$ avec $\omega_{p}=2 \pi c / 0,159 \times 10^{6} \mathrm{rad} / \mathrm{s}$ et $\gamma=0,0048 \omega_{p}$.

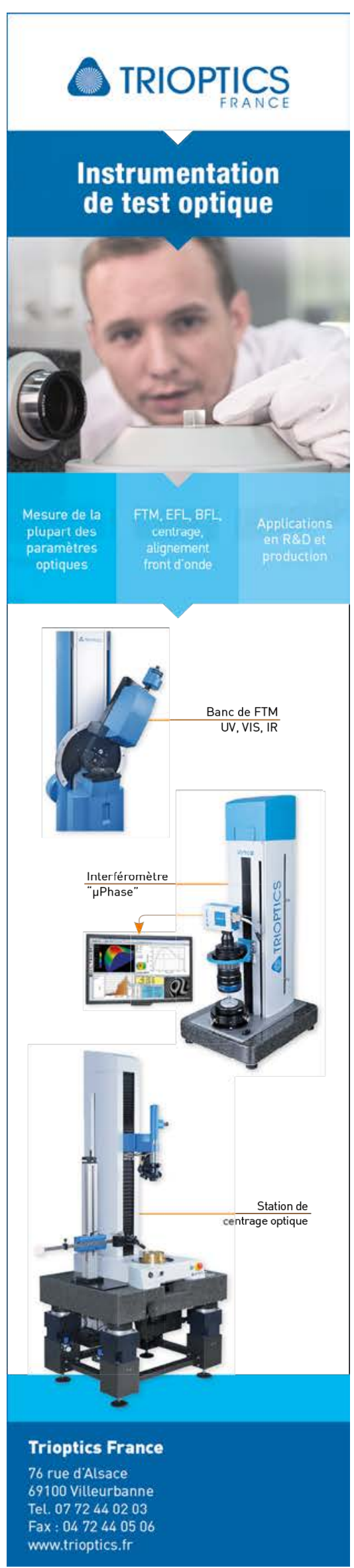


(a)

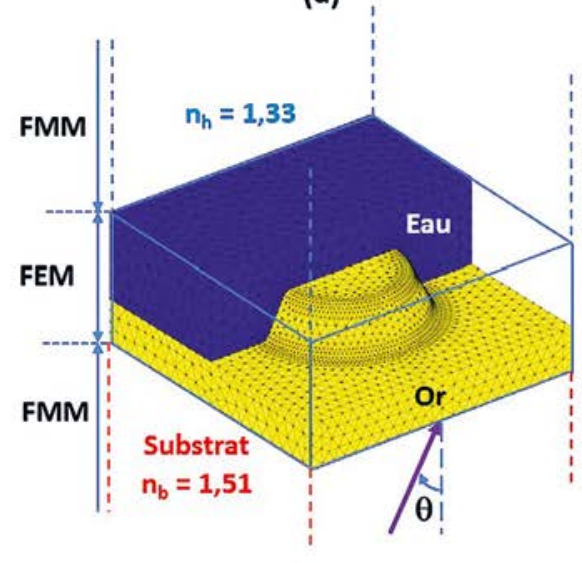

(b)

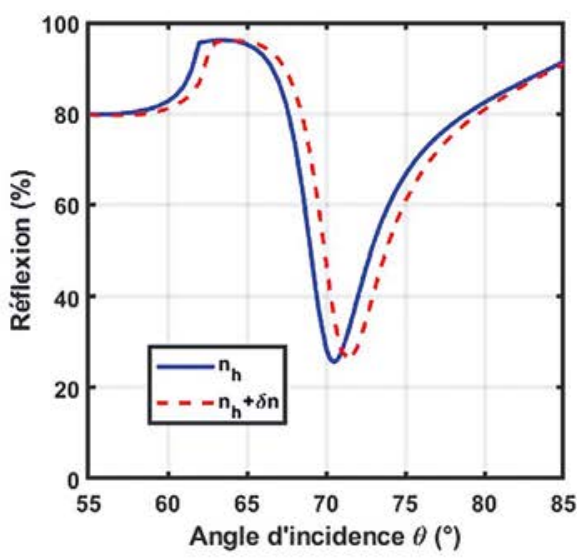

(c)

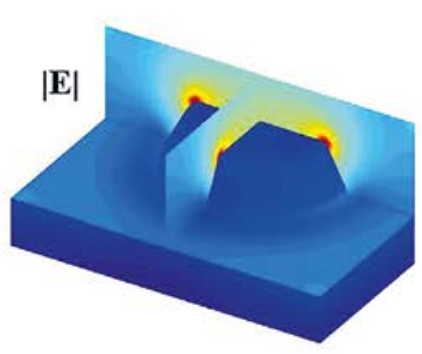

Figure 3.

Calcul des propriétés optiques d'une biopuce à l'aide de la méthode hybride FEM/FMM.

(a) Réseau de période $200 \mathrm{~nm} \times 200 \mathrm{~nm}$ formé par des nanocônes en or tronqués avec des bords arrondis de hauteur $30 \mathrm{~nm}$, de rayon $50 \mathrm{~nm}$ à mi-hauteur, et déposés sur une couche d'or d'épaisseur 30 nm, le tout reposant sur un substrat de BK7.

(b) Réflectivité de la nanostructure avec et sans variation d'indice $\delta n=0,01$ du superstrat (eau avec et sans molécules à détecter) pour une longueur d'onde égale à $850 \mathrm{~nm}$. La structure est éclairée depuis le substrat avec une onde plane faisant un angle $\Theta$ avec la verticale.

(c) Module du champ électrique à la résonance.

\section{"Chaque méthode possède sespropres spécificités, ses avantages et ses inconvénients, mais toutes font face au même défi majeur: calculer le champ électromagnétique au voisinage d'une interface entre un matériau diélectrique et un matériau au caractère métallique prononcé"}

nombreux codes « faits maison » en accès libre sur Internet.

La figure 2 illustre la difficulté du calcul numérique d'une structure présentant de très fortes variations du champ ainsi que l'ajout de transformations de coordonnées dans la FMM pour améliorer la convergence du calcul. La structure est un réseau bidimensionnel de bâtonnets d'or déposés sur un substrat de silice qui présente une résonance d'absorption dans l'infrarouge (figure $2 d$ ). Une coupe horizontale du champ électrique calculé avec la FMM (figure 2b) montre les variations très rapides du champ au niveau des interfaces air/ or ainsi que les singularités dues aux arrêtes. La figure $2 c$ montre que l'ajout d'une transformation de coordonnées réelle permet de dilater l'espace au voisinage des interfaces métalliques et de rendre les variations du champ plus lentes et donc plus faciles à représenter numériquement $[9,10]$. La figure $2 e$ illustre la nette amélioration de convergence qui en résulte.

\section{EXEMPLE DE COUPLAGE DE DEUX MÉTHODES NUMÉRIQUES : MÉTHODE HYBRIDE FEM/FMM}

Pour s'affranchir de certains inconvénients d'une méthode numérique sans en créer de nouveaux, il peut être intéressant de coupler deux méthodes différentes. C'est ainsi qu'a été proposée une méthode hybride associant la méthode des éléments finis (FEM) avec la FMM [11]. L'objectif était de profiter des avantages de la FEM pour traiter des géométries curvilignes complexes tout en conservant le bénéfice de l'intégration analytique de la FMM dans toutes les zones où la géométrie est simple. Cette méthode hybride est particulièrement adaptée aux structures composées de nanoparticules de forme complexe (traitées avec la FEM) inclues dans un empilement de couches homogènes éventuellement plus épaisses que la longueur d'onde (traitées avec la FMM). Les zones où le calcul est fait avec la FMM sont raccordées avec celles où le calcul est fait avec la FEM (figure $3 a$ ) en utilisant la transformée de Fourier des composantes continues des champs calculés avec les éléments finis sur les faces supérieure et inférieure du maillage.

Pour illustrer la méthode hybride, considérons une biopuce formée par une couche d'or dont la surface supérieure est structurée par un réseau bidimensionnel de nanocônes tronqués présentant des bords arrondis et un flanc incliné (figure 3). 
Ce type de biopuce permet d'identifier la présence d'éléments dans l'eau, une protéine par exemple, grâce au décalage de la résonance induit par une variation d'indice $\delta$. La partie structurée et son environnement immédiat sont discrétisés par la FEM et le reste de la structure est modélisé par la FMM (figure $3 a$ ). Cette méthode permet de calculer à la fois les ordres de diffraction et la distribution du champ dans la cellule de calcul. Le modèle hybride garantit généralement une meilleure convergence et un temps de calcul réduit.

\section{CONCLUSION}

Les méthodes numériques permettant de calculer les propriétés optiques de micro et nanostructures sont nombreuses et variées. Nous avons essentiellement discuté de structures photoniques périodiques mais certaines méthodes peuvent être étendues à des structures non-périodiques en remplaçant les conditions aux limites périodiques par des couches parfaitement adaptées (perfectly matched layers, PMLs). C'est le cas des méthodes génériques telles que la FDTD ou les éléments finis mais également de la méthode modale de Fourier dont l'extension au cas apériodique a été proposée au début des années 2000 [9,12]. Dans le cas d'assemblages d'un grand nombre de nanostructures dont l'extension totale est grande devant la longueur d'onde, les calculs restent aujourd'hui très difficiles à cause de la coexistence de plusieurs échelles différentes. Cela est d'autant plus vrai pour des systèmes désordonnés qui sont de plus en plus étudiés pour de nombreuses applications.

\section{RÉFÉRENCES}

[1] A. Taflove, S. Hagness, Computational Electrodynamics: The Finite-Difference Time-Domain Method, $3^{\text {rd }}$ edn. (Artech House, Norwood, MA, 2005)

[2] A. Bossavit, Computational Electromagnetism, Variational Formulations, Complementarity, Edge Elements (Academic Press, 1998)

[3] E. Popov, Gratings: Theory and Numeric Applications, $2^{\text {nd }}$ edn. (2014)

[4] J. van Bladel, Annales des Télécommunications 38, 115 (1983)

[5] K. Knop, J. Opt. Soc. Am. 68, 1206 (1978)

[6] P. Lalanne, G. M. Morris, J. Opt. Soc. Am. A 13, 779 (1996)

[7] G. Granet, B. Guizal, J. Opt. Am. A 13, 1019 (1996)

[8] L. Li, J. Opt. Soc. Am. A 13, 1870 (1996)

[9] J.-P. Hugonin, P. Lalanne, J. Opt. Soc. Am. A 22, 1844 (2005)

[10] G. Granet, J. Opt. Soc. Am. A 16, 2510 (1999)

[11] J.-P. Hugonin et al., Opt. Lett. 33, 1590 (2008)

[12] E. Silberstein et al., J. Opt. Soc. Am. A 18, 2865 (2001)

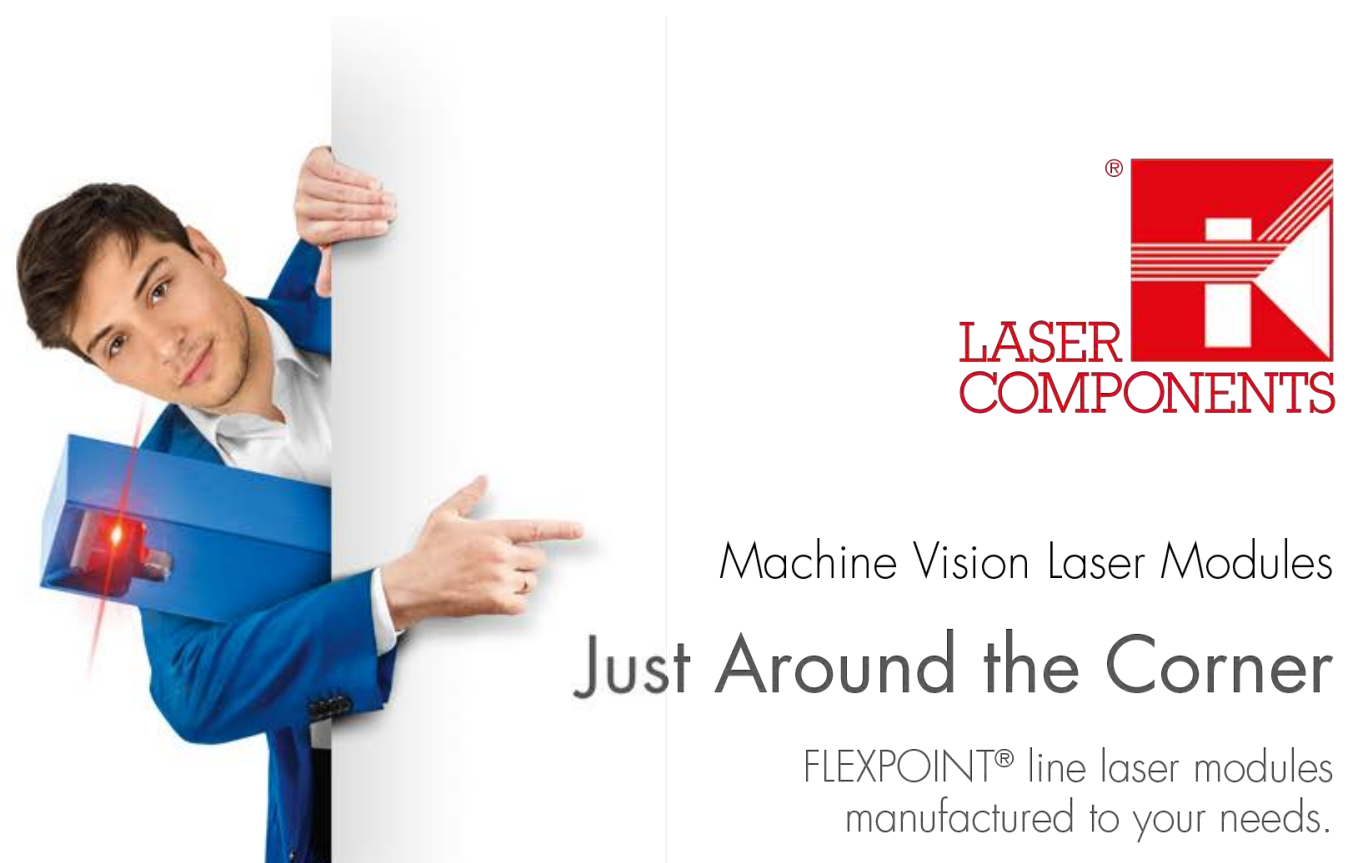

www.lasercomponents.fr 